EPJ manuscript No.

(will be inserted by the editor)

\title{
Non-classical behavior in multimode and disordered transverse structures in OPO
}

\section{Use of the $Q$ representation}

\author{
Roberta Zambrini ${ }^{1}$, Stephen M. Barnett ${ }^{2}$, Pere Colet ${ }^{1}$, and Maxi San Miguel ${ }^{1}$ \\ 1 Instituto Mediterráneo de Estudios Avanzados, IMEDEA (CSIC-UIB), Campus Universitat Illes Balears, E-07071 Palma de \\ Mallorca, Spain. http://www.imedea.uib.es/PhysDept/ \\ 2 Department of Physics, University of Strathclyde, Glasgow G4 0NG, United Kingdom
}

Received: date / Revised version: date

\begin{abstract}
We employ the Q representation to study the non-classical correlations that are present from below to above-threshold in the degenerate optical parametric oscillator. Our study shows that such correlations are present just above threshold, in the regime in which stripe patterns are formed, but that they also persist further above threshold in the presence of spatially disordered structures.

PACS. 42.50.Lc Quantum fluctuations, quantum noise, and quantum jumps - 42.50.Dv Nonclassical field states; squeezed, antibunched, and sub-Poissonian states; operational definitions of the phase of the field; phase measurements - 42.65.Sf Dynamics of nonlinear optical systems; optical instabilities, optical chaos and complexity, and optical spatio-temporal dynamics
\end{abstract}

\section{Introduction}

Quantum correlations in transverse spatial patterns of optical systems have been much studied in recent years. This is because of their fundamental relevance as a macroscopic quantum phenomena in spatially extended systems, and also because of possible applications for quantum image processing [1, 2, 3, 4]. In this context a prototype system studied is the Optical Parametric Oscillator (OPO). Pattern formation in OPO was predicted long ago [5], and there are recent experimental observations of spatial mode interaction and pattern formation 6].

Quantum correlations in the OPO are often understood in terms of the basic process of emission of twin signal photons in parametric down conversion of a pump photon [7, 8]. Below, but close to the threshold for parametric oscillation, any critical transverse mode $\mathbf{k}_{\mathbf{c}}$ is weakly damped. The ring with radius $\left|\mathbf{k}_{\mathbf{c}}\right|$ seen in the far field is a noisy precursor [9] of the wave-number to be selected above threshold. Spatial correlations have been studied in this regime showing interesting non-classical behavior [11. This is referred in the literature as a quantum image [10]. In particular, the intensity difference between any two opposite modes with radius $\left|\mathbf{k}_{\mathbf{c}}\right|$ in the far field is subpoissonian. This is one manifestation of quantum entanglement of the modes 12 . From a technical point of view, correlations in the regime below threshold can be calculated analytically within linear approximations and using the

Send offprint requests to: roberta@imedea.uib.es
Wigner representation [13. Above, but relatively close to threshold, a stripe pattern appears (Sect. 3) due to the interference of two selected signal modes of opposite critical transverse wavenumber [14]. Again, this result can be understood as a macroscopic consequence of the microscopic process of twin photon emission. Close to threshold this phenomenon is studied with models which consider only a small number of modes; only the pump mode and the critical ones are considered. The calculation is based on a linearization approximation and the use of the Wigner representation. At threshold or extremely close to threshold, critical fluctuations would invalidate a linearized calculation, but a few mode approximation, such as those considered in 15, could be appropriate.

Quite a different situation exists when multimode nonlinear dynamics have to be considered. Some results have been obtained in the context of multimode interaction in linear approximations in other systems [16, 17, 18. However, a full multimode nonlinear description is needed well above threshold where the emission of the critical modes necessarily stimulates spatial harmonics in both the pump and the signal, and undamped modes associated to spatial broken symmetries play an important role. This is also the case in the convective regime caused by walk-off, where a noise sustained pattern appears with a broad (multimode) spectrum and amplified nonlinear fluctuations [19, 20. In these situations two relevant questions have to be addressed. First, from the conceptual point of view one might ask if the quantum correlations are degraded by the 
subsidiary nonlinear processes of multimode competition. In particular, can the twin beams that appear close to threshold be incoherently depleted by other processes? If, however, quantum effects persist, then the simple explanation in terms of emission of pairs of twin photons needs to be revised. Secondly, and from a technical point of view, new calculation techniques or approximations that go beyond linearized approximations need to be developed.

We have previously examined these questions using a time-dependent parametric approximation 20,21]. We concluded that quantum correlations were strongly suppressed in the noise-dominated convective regime [20]. However, in the regime of absolute instability we found that non-classical correlations between the $\pm k_{c}$ signal beams persists in spite of nonlinear interactions with the pump and with higher order harmonics of the signal 21]. This is still true in cases in which the mean intensity of the two critical signal beams is different because of walk-off [21]. Two limitations of our time dependent parametric approximation, which is well suited in the convective regime, are that fluctuations in the pump are neglected and that its validity is restricted to values relatively close to threshold.

As an alternative approach we propose, in this paper, the use of the Q-representation and its associated nonlinear Langevin equations for c-number complex fields. With this method we can study quantum spatial correlations in the DOPO without any linearization, few mode approximation or time-dependent parametric approximation, for values of the pump less than twice its threshold value. In particular we can reach high pump values for which the stationary classical solution for the signal field is not a stripe pattern, but rather a homogeneous state with one of two preferred phases. Spatial coexistence of domains of these two homogeneous solutions separated by domain walls gives rise to spatially disordered patterns (seemingly chaotic). These have a broad spectrum in the far field for both the pump and the signal field. This structure cannot be described, not even as a first approximation, as the interference of two twin beams of opposite transverse critical wavenumber. This is because of other cascading processes coupling many different modes and frequencies. Nevertheless, we find that any $\pm k$ pair of modes in the far field that lie within the active range of the broad spectrum show non-classical correlations. This seems to indicate that the basic process of parametric down conversion is behind correlations found even in these very complicated spatial structures.

The paper is organized as follows. The quantum formulation of the problem is presented in Sect. 2, where phase space methods are briefly compared (Sect. 2.1) and Langevin equations for the $\mathrm{Q}$ representation are introduced (Sect. 2.2). In Sect. 3 we briefly review relevant classical results concerning the instabilities to pattern formation and to homogeneous solutions well above threshold. Our general aim is to study the quantum properties of the correlations in regimes that have not been previously studied. These include the critical point in presence of a multimode interaction (Sect. (4), and the above threshold region in presence of complex patterns (Sect. [1). We find signatures of entanglement of the beams in the presence of stripes influenced by the presence of higher harmonics (Sect. 5.1). We show that such entanglement is not only associated with a stripe pattern, but it is present also in spatially disordered structures (Sect. 5.2). Finally Sect. 6 is devoted to concluding remarks.

\section{Quantum formulation of DOPO dynamics}

To describe the intracavity dynamics in a DOPO we introduce the boson spatial modes $\hat{A}_{0}(\mathbf{x}, t)$ and $\hat{A}_{1}(\mathbf{x}, t)$, respectively at the pump frequency $2 \omega$, and signal frequency $\omega$, and satisfying standard equal-time commutation relations 13.

$$
\left[\hat{A}_{i}(\mathbf{x}, t), \hat{A}_{j}^{\dagger}\left(\mathbf{x}^{\prime}, t\right)\right]=\delta_{i j} \delta\left(\mathbf{x}-\mathbf{x}^{\prime}\right), \quad i, j=0,1
$$

Here $\mathbf{x}$ denotes the transverse coordinate(s). A Hamiltonian operator describes the interaction between these modes in the non-linear medium. The intracavity fields constitute an open device [22,23], modeled within a statistical approach in the Schrödinger picture by a Master equation. In Sect.2.1 we review the Master equation of a DOPO. We then report on the possible phase-space descriptions, introducing the Q-representation and the associated Langevin equations (Sect.2.2).

\subsection{Master equation and phase space descriptions}

The intracavity dynamics of our open system is described by a Master equation for the reduced density operator $\hat{\rho}$ [22, 23]:

$$
\frac{\partial \hat{\rho}}{\partial t}=\frac{1}{i \hbar}[\hat{H}, \hat{\rho}]+\hat{\Lambda} \hat{\rho} .
$$

We consider a plane one-sided cavity, hence the Liouvillian accounting for dissipation through the partially reflecting mirror is given by

$$
\hat{\Lambda} \hat{\rho}=\sum_{i=0,1} \gamma_{i} \int d^{2} \mathbf{x}\left\{\left[\hat{A}_{i}(\mathbf{x}), \hat{\rho} \hat{A}_{i}^{\dagger}(\mathbf{x})\right]+\left[\hat{A}_{i}(\mathbf{x}) \hat{\rho}, \hat{A}_{i}^{\dagger}(\mathbf{x})\right]\right\}
$$

The Hamiltonian operator, expressed as a function of fields operators $\hat{A}_{0}(\mathbf{x}, t)$ and $\hat{A}_{1}(\mathbf{x}, t)$, is:

$$
\hat{H}=\hat{H}_{0}+\hat{H}_{i n t}+\hat{H}_{e x t}
$$

where 24

$$
\hat{H}_{0}=\hbar \int d^{2} \mathbf{x} \sum_{i=0,1}\left[\gamma_{i} \hat{A}_{i}^{\dagger}(\mathbf{x})\left(\Delta_{i}-a_{i} \nabla^{2}\right) \hat{A}_{i}(\mathbf{x})\right]
$$

describes free propagation of fields in the cavity,

$$
\hat{H}_{e x t}=i \hbar \int d^{2} \mathbf{x} E\left[\hat{A}_{0}^{\dagger}(\mathbf{x})-\hat{A}_{0}(\mathbf{x})\right]
$$


is due to the interaction with the external pump $E$, which we choose to be real, and

$$
\hat{H}_{\text {int }}=i \hbar \frac{g}{2} \int d^{2} \mathbf{x}\left[\hat{A}_{0}(\mathbf{x}) \hat{A}_{1}^{\dagger 2}(\mathbf{x})-\hat{A}_{0}^{\dagger}(\mathbf{x}) \hat{A}_{1}^{2}(\mathbf{x})\right]
$$

is the interaction term between first and second harmonic.

Density operators in state space can be mapped to quasi-probability distribution densities on phase space. These can be used to calculate ensemble averages of operators in defined orderings 25,26. Using this "quantum-toclassical" correspondence, Eq. (2) can be converted into an equation of motion for a quasi-probability distribution in the phase-space of radiation fields $\alpha_{i}(\mathbf{x})$, associated with the operators $\hat{A}_{i}(\mathbf{x})$.

The presence of non-linearities leads to a functional differential equation for the quasi-probability that is not of the Fokker-Planck type, leading to difficulties in obtaining solutions 27]. The offending term for the system of interest is $\hat{H}_{\text {int }}$, which gives a functional term of the form

$$
\begin{aligned}
& {\left[\hat{A}_{0}(\mathbf{x}) \hat{A}_{1}^{\dagger}(\mathbf{x})-h . c ., \hat{\rho}\right] \Longleftrightarrow\left(s \alpha_{0} \frac{\delta^{2}}{\delta \alpha_{1}^{2}}+\right.} \\
& \left.\frac{1-s^{2}}{4} \frac{\delta^{3}}{\delta \alpha_{1}^{2} \delta \alpha_{0}^{*}}+\frac{\delta}{\delta \alpha_{0}} \alpha_{1}^{2}-2 \alpha_{0} \alpha_{1}^{*} \frac{\delta}{\delta \alpha_{1}}+\text { c.c. }\right) W_{s} .
\end{aligned}
$$

Here $s$ denotes the operator ordering selected. We see that third order derivatives appear in the temporal evolution of the Wigner representation $(s=0)$. The approximate equations obtained simply dropping these third order terms constitute the basis of stochastic electrodynamics 28]. This approach works well in linear regimes, in which the Wigner distribution satisfies a genuine Fokker-Planck equation. In particular, in the DOPO below the threshold of signal generation the intensity of the signal is of the order of the quantum noise, while the pump has a macroscopic mean value, so that its fluctuations can be neglected. With the assumption of a classical undepleted pump, the Hamiltonian that describes the quantum dynamics of the signal is quadratic and the Langevin equation [27] - equivalent to the Fokker Planck equation for Wigner representation - can be analytically solved [13]. Recent investigations have shown the limits of this stochastic electrodynamics in reproducing quantum higher-order moments [29]. The same type of approximation is generally possible above threshold, linearizing around a pattern solution [16]. Due to the in-homogeneity of the reference state, moreover, only semi-analytical or numerical simulations can be provided. Above threshold great care has to be taken if the system is translational invariant (flat mirrors and homogeneous transversal pump profile). The pattern solution breaks the translational symmetry and therefore there is a Goldstone mode which is neutrally stable [16]. Noise excites this mode, giving diffusion of the phase which fixes the position of the pattern [16]. Moments involving such big fluctuations cannot be correctly described within a linearized treatment in the fields amplitudes. In particular, such an approach leads to unphysically divergent quadrature correlations, although correct results can be obtained for the intensity correlations 18].
Another well-known quasi-probability is the $\mathrm{P}_{+}$representation [30, consisting in the extension of the normal ordered $\mathrm{P}$ representation over a doubled phase space [31]. The $\mathrm{P}$ representation $(s=1$ in Eq. (8) $)$ suffers of negative diffusion in problems of interest, but the $\mathrm{P}_{+}$generally gives good results, with the advantage of the possibility to obtain immediately also the moments outside the cavity. However, in some systems this doubling phase-space technique has shown divergent trajectories, as reviewed in Ref. [32]. In particular, there are regimes in extended systems -like the convective regime [20]- in which the presence of large fluctuations around the unstable reference state would result in diverging trajectories in the $\mathrm{P}_{+}$and alternative methods are needed.

In this paper we employ the Q representation corresponding to anti-normal ordering of field operators. The most important property of this representation is that it satisfies the requirements for a true probability distribution. In fact the Q-representation may be defined as the diagonal matrix elements of the density operator in the space of coherent states

$$
Q\left(\alpha_{0}, \alpha_{1}\right)=\frac{1}{\pi}<\alpha_{0}, \alpha_{1}|\hat{\rho}| \alpha_{0}, \alpha_{1}>
$$

and so is both positive and bounded [26]. Due to the over completeness of the coherent states ensemble, the definition (8) uniquely determines the density operator $\hat{\rho}$. Physically this representation, resulting from a Gaussian convolution of the Wigner representation, corresponds to simultaneous measurements of orthogonal quadratures, as limited by the Heisenberg principle, in a eight-port homodyne detector [33]. From Eq. (7) (with $s=-1$ ) we observe that the Q-representation suffers of negative diffusion. Unlike the Wigner function, however, the Q function is always positive and well-behaved. The possibility to obtain a positive solution in presence of a negative diffusion [34 lies in the presence of a restricted ensemble of initial conditions, that cannot be arbitrarily narrow. In other words, not all mathematical forms for the Q function correspond to physical states. The evolution of a physical state - corresponding to an hermitian density operator - in the $\mathrm{Q}$ representation will always be positive [26, 35]. We should note that a $\mathrm{Q}$ representation with a doubled phase-space has been proposed in order to deal with negative diffusion [36]. This has been shown to give good results in some non-linear quantum systems [37].

In the next section we investigate the possibility to use the Q-representation for devices consisting on a cavity filled with a $\chi^{2}$ medium, as in the OPO and Second Harmonic Generation (SHG).

\subsection{Langevin equations in Q-representation}

The evolution equation of the functional Q for our model (described above) is:

$$
\frac{\partial Q\left(\alpha_{0}, \alpha_{1}\right)}{\partial t}=\int d^{2} \mathbf{x}\left\{-\left(\frac{\delta}{\delta \alpha_{0}(\mathbf{x})} V_{0}+\frac{\delta}{\delta \alpha_{1}(\mathbf{x})} V_{1}+c . c .\right)\right.
$$




$$
\begin{aligned}
& +\int d^{2} \mathbf{x}^{\prime}\left[2 \gamma_{0} \frac{\delta^{2}}{\delta \alpha_{0}(\mathbf{x}) \delta \alpha_{0}^{*}\left(\mathbf{x}^{\prime}\right)}+2 \gamma_{1} \frac{\delta^{2}}{\delta \alpha_{1}(\mathbf{x}) \delta \alpha_{1}^{*}\left(\mathbf{x}^{\prime}\right)}+\right. \\
& \left.\left.\frac{1}{2}\left(-g \alpha_{0} \frac{\delta^{2}}{\delta \alpha_{1}(\mathbf{x}) \delta \alpha_{1}\left(\mathbf{x}^{\prime}\right)}+c . c\right)\right]\right\} Q\left(\alpha_{0}, \alpha_{1}\right),
\end{aligned}
$$

where the drift terms are

$$
\begin{aligned}
& V_{0}=-\gamma_{0}\left[\left(1+i \Delta_{0}\right)-i a_{0} \nabla^{2}\right] \alpha_{0}(\mathbf{x}, t)-\frac{g}{2} \alpha_{1}^{2}(\mathbf{x}, t)+E \\
& V_{1}=-\gamma_{1}\left[\left(1+i \Delta_{1}\right)-i a_{1} \nabla^{2}\right] \alpha_{1}(\mathbf{x}, t)+g \alpha_{0}(\mathbf{x}, t) \alpha_{1}^{*}(\mathbf{x}, t) .
\end{aligned}
$$

If the diffusion term is positive then our evolution equation is a bona-fide Fokker-Planck equation. In the other case this equation doesn't describe an ordinary diffusion process. For equation (9) the diffusion term is positive if

$$
\left|\alpha_{0}(\mathbf{x}, t)\right|<\frac{2 \gamma_{1}}{g} .
$$

The modulus of the stationary field at threshold takes the value $\left|A_{0}^{\text {thr }}\right|=\gamma_{1} / g$ (see Sect. 3). This means that the condition (10) corresponds to pump trajectories taking values that are less than twice the threshold value. Staying in a region far from the limit (10) - we are considering $A_{0}^{s t} \leq 1.5 A_{0}^{t h r}$ - an extremely large fluctuation in a trajectory would be necessary in order to lose the positiveness of the diffusion. Clearly these trajectories have a negligible probability to appear, and never appeared in our simulations. For these reasons, the approximation we propose is to study Langevin equations related to the Fokker-Planck equation given by (9) and (10), neglecting any trajectories that would make negative the diffusion term. Clearly the condition (10) does not depend on the frequency at which the system is pumped. For this reason the method is suitable, and has been already successfully used, to describe non-linear fluctuations in stripe patterns in SHG in a regime of pump values limited by Eq. (10) 38].

From Eqs. (9-10), with the scaling (D is the transversal dimensionality of the system)

$$
\begin{aligned}
& \gamma_{0}=\gamma_{1}=\gamma, \quad a_{0}=a_{1} / 2=a, \\
& t^{\prime}=\gamma t, \quad \mathbf{x}^{\prime}=\frac{\mathbf{x}}{\sqrt{a}}, \\
& A_{i}^{\prime}=\frac{g}{\gamma} A_{i}, \quad E^{\prime}=\frac{g}{\gamma^{2}} E, \quad \epsilon_{i}^{\prime}=\frac{g}{\gamma^{3 / 2} a^{D / 4}} \epsilon_{i},
\end{aligned}
$$

we obtain the equations:

$$
\begin{aligned}
\partial_{t} \alpha_{0}(\mathbf{x}, t)= & -\left[\left(1+i \Delta_{0}\right)-i \nabla^{2}\right] \alpha_{0}(\mathbf{x}, t)+E- \\
& \frac{1}{2} \alpha_{1}^{2}(\mathbf{x}, t)+\sqrt{\frac{2}{a}} \frac{g}{\gamma} \xi_{0}(\mathbf{x}, t) \\
\partial_{t} \alpha_{1}(\mathbf{x}, t)= & -\left[\left(1+i \Delta_{1}\right)-2 i \nabla^{2}\right] \alpha_{1}(\mathbf{x}, t)+ \\
& \alpha_{0}(\mathbf{x}, t) \alpha_{1}^{*}(\mathbf{x}, t)+\sqrt{\frac{2}{a}} \frac{g}{\gamma} \xi_{1}(\mathbf{x}, t) .
\end{aligned}
$$

The condition (10) in the new variables is

$$
\left|\alpha_{0}(\mathbf{x}, t)\right|<2 .
$$

We solve these Langevin equations by numerical simulation, neglecting any trajectories that do not satisfy the condition (14), should these occur. $\xi_{0}$ is a white Gaussian noise with non-vanishing moment:

$$
\left\langle\xi_{0}(\mathbf{x}, t) \xi_{0}^{*}\left(\mathbf{x}^{\prime}, t^{\prime}\right)\right\rangle=\delta\left(\mathbf{x}-\mathbf{x}^{\prime}\right)\left(t-t^{\prime}\right)
$$

The signal noise $\xi_{1}$ results to be phase sensitive, due to the presence of diagonal terms in the diffusion matrix of Eq. (9). Moreover this noise is multiplicative, depending on the value of the pump field. However due to the form of $\hat{H}$ (quadratic in $\hat{A}_{1}$ and linear in $\hat{A}_{0}$ ), these equations have the same formal expression in the Ito or Stratonovich interpretations [27].

The phase sensitive multiplicative noise $\xi_{1}(x, t)$ can be written as

$$
\begin{aligned}
\xi_{1}(x, t) & =\left[\frac{-\alpha_{0 I}(x, t)}{2 \sqrt{2+\alpha_{0 R}(x, t)}}+\frac{i}{2} \sqrt{2+\alpha_{0 R}(x, t)}\right] \phi(x, t) \\
& +\sqrt{\frac{1-\frac{\left|\alpha_{0}(x, t)\right|^{2}}{4}}{2+\alpha_{0 R}(x, t)}} \psi(x, t)
\end{aligned}
$$

with $\alpha_{0}=\alpha_{0 R}+i \alpha_{0 I}$ and $\phi, \psi$ uncorrelated real white noises in space and time, with variances one. Actually, the diffusion matrix of a Fokker-Plank equation fix only three of the four degrees of freedom in the choice of the real and imaginary parts of the noise term $\xi_{1}$; this depends on the multiplicity of Langevin processes associated with the same Fokker-Planck equation [27]. Hence Eq. (16) corresponds to a particular choice among several possible representations [39].

In the next sections we present the numerical results obtained by simulating Eq. (12) and Eq. (13) with the same integration method of Ref. [16, 38. In particular we consider one transversal dimension $(\bar{D}=1)$ and parameters:

$$
\Delta_{0}=0, \quad \Delta_{1}=-0.18, \quad \frac{g}{\sqrt{a} \gamma}=10^{-4}
$$

with a system size of four critical wavelengths $L=4 \lambda_{c}$, with $\lambda_{c}=\frac{2 \pi}{k_{i}}$ (see Sect. 3).

Eqs. (12 13) are suitable for the study of quantum fluctuations in different regimes - discussed in Sect. 3 from the linear regime below threshold to the multimode regimes quite above threshold. In Sect. 1 we present calculations of quantum correlations below threshold and at the critical point, comparing with analytical results in the linear approximation. In Sect. 5 we consider the above threshold regime, in the situation of regular stripe pattern formation and also when disordered structures are formed (see Fig. 1).

\section{Pattern formation in OPO}

We first review the patterns that arise in the OPO in the classical limit. The phase matched DOPO, where both pump $A_{0}$ and signal $A_{1}$ fields are resonated, is described classically by equations identical to Eqs. (12) and (13) but 
neglecting the noise terms. The classical equations have a trivial homogeneous solution

$$
A_{1}^{s t}=0, A_{0}^{s t}=\frac{E}{1+i \Delta_{0}} .
$$

A linear stability analysis around this solution gives the following dispersion relation for the growth of the signal field perturbations with wave vector $k$ (the pump field $A_{0}$ is always stable) [5]

$$
\lambda_{1}(\mathbf{k})=-1 \pm \sqrt{\left|A_{0}^{s t}\right|^{2}-\left(\Delta_{1}+2 k^{2}\right)} .
$$

For negative signal detunings, the zero homogeneous solution becomes unstable at $E=E_{c}=\sqrt{1+\Delta_{0}^{2}}$. The perturbations with maximum growth rate are those with wave number $\left|k_{c}\right|=\sqrt{-\Delta_{1} / 2}$, and a pattern with this wave number is formed at threshold [5]. For positive signal detunings the zero homogeneous solution is stable for $E<E_{c}=\sqrt{\left(1+\Delta_{0}^{2}\right)\left(1+\Delta_{1}^{2}\right)}$. In this case the instability takes place at zero wave number leading to a non zero homogeneous solution [40]

$$
\begin{aligned}
& A_{1}^{s t}= \pm \sqrt{\frac{E\left|A_{1}^{s t}\right|^{2}}{\left(1+i \Delta_{0}\right)\left(1+i \Delta_{1}\right)+\left|A_{1}^{s t}\right|^{2}}} \\
& A_{0}^{s t}=\frac{E\left(1+i \Delta_{1}\right)}{\left(1+i \Delta_{0}\right)\left(1+i \Delta_{1}\right)+\left|A_{1}^{s t}\right|^{2}} .
\end{aligned}
$$

There are two equivalent solutions for the signal field with a $\pi$ phase difference.

In this paper we will consider the case of zero pump detuning $\left(\Delta_{0}=0\right)$ and negative signal detuning $\left(\Delta_{1}<0\right)$, in which stripe pattern arises at threshold $\left(E_{c}=1\right)$. Increasing further the pump the nonzero homogeneous solutions (20) become stable. In fact, we observe they are stable for pump values around $E=1.1$. This is lower than the value at which the stripe pattern becomes linearly unstable 41, 42. Numerical studies in this regime find multistability between the stripe pattern, the two nonzero homogeneous solutions and several irregular spatially modulated solutions. The latter are formed by fronts with oscillatory tails connecting the two equivalent homogeneous solutions. In systems with two spatial dimensions, there are also coexisting labyrinthine patterns. In this case $(D=2)$ both the irregular spatially modulated solutions and the labyrinthine patterns cease to exist at the modulational instability of a flat front connecting the two homogeneous solutions 42, 43. Here we consider systems with only one spatial dimension for which such an instability does not exist. This means that the regime of multistability in parameter space is much larger. The irregular spatially modulated solutions found in this system are an example of frozen chaos as described in Ref. 44. In that case the interaction of two distant fronts can be described by a potential with several wells which become progressively deeper as the distances between the fronts decreases. The OPO cannot be described in terms of such potentials, but numerical studies reveal equilibrium distances whenever the maxima (or the minima) of the local oscillations of the front overlap with each other [41, 42].

\section{Quantum correlations below and at threshold}

The spatiotemporal dynamics of the signal field is shown in Fig. 1 for two relevant values of the pump, below but near to threshold (quantum images regime), $E=0.999$ and at threshold $(E=1)$. The far field $(\mathrm{FF})$ shows strong fluctuations dominated by the critical wave-vector: in Sects. (4.1.4.2) we discuss the quadratures and intensity quantum correlations of these modes.

\subsection{Quadrature correlations}

The direction in which quadrature squeezing appears is determined by the eigenfunction $V_{ \pm}(k,-k)$ of the linear problem $\partial_{t} V_{ \pm}(k,-k)=\lambda_{ \pm}(k) V_{ \pm}(k,-k)$, as reported in Ref. [20]:

$$
\begin{aligned}
& V_{ \pm}(k,-k)=e^{i \Phi_{ \pm}} \delta A_{1}(k) \pm \delta A_{1}^{*}(-k) \\
& e^{i \Phi_{ \pm}(k)}=\mp \frac{i \Delta_{1}+2 i k^{2} \mp \sqrt{\left|A_{0}^{s t}\right|^{2}-\left(\Delta_{1}+2 k^{2}\right)^{2}}}{A_{0}^{s t}} .
\end{aligned}
$$

The solution $V_{+}(k,-k)$ gives the direction of amplification of fluctuations, while fluctuations are damped for $V_{-}(k,-k)$, giving rise to quadrature squeezing. In particular, for the critical wave-vector $k_{c}$ and for our choice of parameter (real $A_{0}^{s t}$ ) we obtain $V_{ \pm}\left(k_{c},-k_{c}\right)=\delta A_{1}\left(k_{c}\right) \pm$ $\delta A_{1}^{*}\left(-k_{c}\right)$. Therefore, the largest squeezing at threshold will be in the difference of real parts and the sum of imaginary parts of the field for wave-numbers $k_{c}$ and $-k_{c}$.

We define the real quadrature operator:

$$
\hat{X}(k)=\hat{A}_{1}(k)+\hat{A}_{1}^{\dagger}(k)
$$

and the quadrature superpositions

$$
\begin{aligned}
& \hat{X}_{-}(k)=\hat{X}(k)-\hat{X}(-k) \\
& \hat{X}_{+}(k)=\hat{X}(k)+\hat{X}(-k),
\end{aligned}
$$

corresponding, respectively, to damped and undamped quantities at threshold.

Below threshold, within a linearization approximation [13], the normal-ordered variances normalized to the shot noise $\left(\mathcal{N}_{X}\right)$ 45] are:

$$
\begin{aligned}
& \frac{<:\left(\hat{X}_{-}\left(k_{c}\right)\right)^{2}:>}{\mathcal{N}_{X}}=\frac{-E}{1+E} \\
& \frac{<:\left(\hat{X}_{+}\left(k_{c}\right)\right)^{2}:>}{\mathcal{N}_{X}}=\frac{E}{1-E} .
\end{aligned}
$$

These quantities coincide with the variances since the mean values are zero: $<\hat{X}_{ \pm}(k)>=0$. The normal ordering allows us to immediately identify non-classical features associated with squeezing such as negative variances. Eq. (26) shows an increasing degree of squeezing, approaching the value -0.5 at threshold. In Fig. 2 theoretical predictions and numerical results are shown to be in good agreement, confirming the validity of Eqs. (12 13) below threshold. 
On the other hand Eq. (27) is always positive indicating that the the fluctuations in the direction of instability are essentially classical and larger than those found for a coherent state. In Fig. 3 we show the agreement between theoretical predictions and numerical results for the undamped quadrature, even as close as $1 \%$ oto threshold. The limits of the linear treatment, discussed above, are now evident in the divergence of Eq. (27) for $E \rightarrow 1$. In contrast, numerical simulation of the nonlinear Eqs. (12 13) gives the expected saturation at the critical point, at a value which depends on the noise level.

\subsection{Intensity correlations}

We can find non-classical features in the intensities of the twin beams by evaluating the normal-ordered variance in the difference of the two intensities:

$$
\mathcal{V}(k)=\frac{\left\langle:\left[\delta \hat{N}_{1}(k)-\delta \hat{N}_{1}(-k)\right]^{2}:\right\rangle}{\mathcal{N}_{N}(k)},
$$

normalized to the corresponding shot noise value $\mathcal{N}_{N}(k)$. This value is proportional to the sum of the intensities of the two beams with wavevectors $\pm k$. Negative values of $\mathcal{V}$ indicate sub-Poissonian statistics for the intensity difference of the two signal beams at $\pm k$ 21. In a linear analytical treatment below threshold $\mathcal{V}(k)=-0.5$, independently of the pump intensity and of the wave-vector [13,21. In other words the normalized intensity correlations, Eq. (28), do not show a non-classical behavior which is stronger for the critical wave vector or at the critical point. This is in contrast with the behavior of the quadratures correlations Eqs. (26 27). Nevertheless, the critical conditions are of significant interest because of presence of higher intensities.

The numerical expression of $\mathcal{V}(k)$ for different spatial modes $\left(0<k \leq 5 k_{c}\right)$ is compared, in Fig. \&, with the analytical value -0.5 for pump $E=0.99$, showing good agreement. Small deviations for large wavevectors $k$ can appear numerically due to the smallness of the shot noise to which the variance is normalized; $\mathcal{N}_{N}$ is proportional to the mean intensity of the field, shown also in Fig. 4 .

In Fig. 5 we plot the variance $\mathcal{V}\left(k_{c}\right)$ at the critical wave-vector as a function of the pump $E$ : We obtain good agreement with analytical predictions below threshold.

\section{Quantum correlations above threshold}

The non-linear equations in the Q-representation are used here to study the regime of pattern formation above threshold. These equations improve the time-dependent parametric approximation introduced in Ref. [20], which is well suited to study the convective regime (in presence of walkoff) or the instability point, because the pump fluctuations are disregarded with respect to the signal ones. Eqs.(12) and (13) are valid in a wide region above threshold, and give a complete description of the pump fluctuations.
Slightly above the threshold $(E=1.02)$ the variance of the quadratures superposition Eq. (24) becomes classical. To understand the how the mode dynamics changes when going above threshold, we recall that below threshold the trajectory $\alpha_{1}\left(k_{c}, t\right)$ occupies a circular region centered in zero, in the phase space given by its real and imaginary part. Above threshold the mean amplitude has a macroscopic value increasing with the pump intensity and the distribution of fluctuations in phase and intensity quadratures is rather different, as shown in Fig. Fa. A trajectory for a mode during a larger time would describe a circle in phase space.

The origin of these large fluctuations in the phase quadrature is well known in the theory of single transversemode non-degenerate parametric oscillators (NDOPO) [46]. Due to the diffusion of the difference of the signal and idler phases, the above threshold solution is not stable, and "cannot be analyzed correctly by the assumption of small fluctuations and methods of linearization" 46. The situation is similar to that of the laser above threshold, for which a correct analysis is performed using intensity and phase variables and not linearizing in the diffusing (phase) variable. In Ref. [17] an exact steady-state Wigner function is calculated in the single transverse-mode NDOPO by adiabatically eliminating the pump. The phase diffusion in a mode is evident in the radial symmetry of this distribution.

The single transverse-mode NDOPO is equivalent to a three mode model, describing the extended DOPO near threshold [14] through the relevant fields $\alpha_{1}\left( \pm k_{c}\right)$ and $\alpha_{0}(0)$ [48. The stationary signal is

$$
\begin{aligned}
\alpha_{1}(x, t) & =\alpha_{1}\left(k_{c}\right) e^{i k_{c} x}+\alpha_{1}\left(-k_{c}\right) e^{-i k_{c} x} \\
& =2\left|\alpha_{1}\left(k_{c}\right)\right| e^{i \frac{\theta_{+}+\theta_{-}}{2}} \cos \left(\frac{\theta_{+}-\theta_{-}}{2}\right),
\end{aligned}
$$

with $\alpha_{1}\left( \pm k_{c}\right)=\left|\alpha_{1}\left( \pm k_{c}\right)\right| e^{i \theta_{ \pm}}$. We recognize the effect of the phases $\theta_{ \pm}$in the near field. The sum of these phases fixes the global phase of the signal, locked to the pump, while the arbitrary phase difference fixes the spatial position of the stripe pattern. In continuous systems, where all modes are taken into account, the diffusion of the phase difference can be interpreted as the action of the Goldstone mode [16, that is neutrally stable, giving a continuous translation of the pattern. This is particularly evident in Fig. 1 1 at threshold $(E=1)$.

In a linear treatment below threshold, the fluctuations of $\left\langle\theta_{+}+\theta_{-}\right\rangle$have zero average and are damped (with $\Delta_{0}=0$ ). Increasing the pump we always observe small fluctuations, but the average changes. Fig. Gb shows that the mean value $\left\langle\theta_{+}+\theta_{-}\right\rangle$increases from its zero value with the distance above threshold. This is a non-linear effect due to the feedback of the signal on the pump. Above threshold the average of the non-linear term in the pump equation is not zero, so the pump is no longer real and this induces a phase rotation in the signal. Therefore it should be expected that the strongest quadrature squeezing will be eventually found for a local oscillator phase that depends on this phase rotation. Here, however, we will restrict our attention to the non-classical features associated 
with intensity correlations. In fact this corresponds to a measure of quadrature squeezing as they result from the interference of a local oscillator given by the mean signal field with the squeezed fluctuations of the same mode.

\subsection{Intensity correlations in stripe patterns}

The stripe pattern formed above threshold is due to the interference of signal beams with opposite critical wavevectors. Momentum conservation leads to the entanglement between these signal beams [7, 14,21]. This gives non-classical intensity correlations characterized by $\mathcal{V}=-0.5$.

Increasing the pump intensity we observe excitation of harmonics of the critical wavenumber (compare far field $(\mathrm{FF})$ in Fig. 1 for $E=1$ and for $E=1.1$ ). In Fig. 7 we show the real part of the near field (NF) pattern in the pump and in the signal and the corresponding FF intensities for pumps $E=1.02$ and $E=1.1$. We observe that the odd harmonics are excited in the signal and the even ones in the pump mode, with an exponential decay of energy at higher wavevector modes. The presence of a multimode interaction means that the momentum conservation no longer constrains the intensities of the twin beams, as it does below and at threshold. However, as shown in Ref. [21], we do observe in this regime the symmetry $\left\langle N_{1}(k)\right\rangle=\left\langle N_{1}(-k)\right\rangle$ in intensity averages and quantum correlations between the critical modes survive, as shown in Fig. 5 for $1<E<1.1$. The secondary process of upconversion of pairs of signal photons $+k_{c}$ (or equivalently $\left.-k_{c}\right)$ to form pump photons $+2 k_{c}\left(-2 k_{c}\right)$ does not seem to destroy the quantum correlations between the signal "twin" photons $\left(+k_{c}\right.$ and $\left.-k_{c}\right)$. In principle this process gives an incoherent depletion of the "twin" beams, but probably due to the smallness of this secondary effect, the quantum correlations associated with the fundamental process survives.

The spatial spectrum of the intensity variance $\mathcal{V}$ is plotted in Fig. 8. We observe that at $2 \%$ above threshold the spectrum is similar to the spectrum below threshold (compare with Fig. 4). A reduction of the squeezing is observed (peak at $k=3 k_{c}=0.9$ in Fig. 8), however, corresponding to the appearance of the third harmonic. Increasing the pump to $10 \%$ above threshold we observe an enhancement of the spectral bandwidth in which this reduction of squeezing appears. The third harmonic in the signal is involved in at least two important processes: the down-conversion of the homogeneous pump into twin photons $+3 k_{c}$ and $-3 k_{c}$ and the secondary process of downconversion of the second harmonics $\pm 2 k_{c}$ of the pump into opposite signal photons $\pm 3 k_{c}$ and $\mp k_{c}$. We note that as the Hamiltonian operator is Hermitian, the opposite (upconversion) processes are also allowed. The observed reduction of squeezing can be interpreted as a signature of the mentioned secondary process, in which the pairs signal $+3 k_{c}$ and $-3 k_{c}$ photons are incoherently (not simultaneously) generated and destroyed. In other words, signal modes are depleted independently, taking part in different cascading processes and generating harmonics. The entanglement should be preserved in opposite signal modes for which the fundamental down-conversion process prevails. We have confirmed that opposite spatial modes in the pump field do not show quantum correlations.

We note that the variances $\mathcal{V}$ obtained with the Qrepresentation -after reordering- are in good agreement with the corresponding quantities calculated with the time dependent parametric approximation in the Wigner representation in Ref. 21].

\subsection{Intensity correlations in spatially disordered structures}

With increasing pump intensity, a transition from a modulated pattern to homogeneous solutions takes place 49. Due to the bistability in this regime, different homogeneous solutions can be selected in separated spatial domains (see Fig. 1 for $E=1.5$ ). All solutions presented in Fig. 1 are obtained starting from a modulated initial condition at $k_{c}$. Therefore we are stimulating, with a particular initial configuration, the shape of the final structure. In Fig. 9a we show the stationary configuration for $E=1.5$, in which the most excited mode is $k_{c}$. The disordered character of the structure gives rise to a broad spectrum, in which the other dominant modes are not harmonics of $k_{c}$. The most intense signal modes combine to form pump modes: for example in Fig. 9 a the signal modes $k_{c}=4 \Delta k=0.3$ and $9 \Delta k=0.675$ give the pump mode $13 \Delta k=0.975$ 50. The stationary disordered structure shows the same reflection symmetry $\left\langle N_{1}(k)\right\rangle=\left\langle N_{1}(-k)\right\rangle$, of the stripe pattern considered in Sect. 5.1.

Studying the properties of the quantum fluctuations in this regime, we observe non-classical correlations between the two signal "twin" beams with critical wave-vectors, also in these spatially disordered structures (see Fig. 5 for $1.2 \leq E \leq 1.5)$. This result does not depend on momentum conservation or on the presence of a regular pattern. We have also considered the entanglement properties of modes different from the critical one. In Fig. 10 we show the spatial spectrum of the variance $\mathcal{V}$. As in the case of a regular stripe pattern, analyzed in the previous section, we continue to find quantum correlated twin beams. However there are some interesting differences. The peak in $\mathcal{V}$ now corresponds to a strongly depleted (low mean intensity) signal mode for $k=0.975$. This contrasts with the previous case, where $k=3 k_{c}$ was an excited mode. The most interesting feature is the appearance of a bandwidth of "twin" beams, where the signal field is intense; the correlations become classical for big wave-vectors $(k \gtrsim 1)$, where the signal is depleted more than the pump field, reaching asymptotically the level of coherent states (see dotted grey line in Fig. 10). In conclusion, the "twin"beams quantum correlations persist in disordered structures. This signature of the fundamental down-conversion process is preserved throughout the region of intense signal modes.

The demanding question is how the spectrum of the variance $\mathcal{V}$ is influenced by the shape of the selected spatial structure. In this regime no special character is associated with the critical wave-length periodicity. There- 
fore we consider a stationary state of two domains, obtained from an initial step condition (Fig. 9 b). Also in this case, with a very different stationary state, we observe non-classical intensity correlations in the bandwidth $0<k \lesssim 1$, as shown in Fig. 10 (black line). In this case no peaks appear, suggesting that the presence of modes with reduced squeezing (as the peak for $k=0.975$ observed in the previous structure) depends on the selected spatial structure.

The key point is the relative importance of the fundamental coherent process of twin photon down-conversion, and other incoherent cascading processes, which depends on the spatial configuration chosen by the system. To give more evidence of this statement, we study the variance $\mathcal{V}$ in three different spatial configurations, obtained for $E=1.3$, starting the simulations from noise, from rolls (as in Fig. 9a) and from a step function (as in Fig. 9b). In Fig. 11 we show the spectral variances (a) and the mean FF intensity (b). The domain configurations obtained staring from noise and from a step function give overlapping smooth variances, while two peaks appear when starting from rolls. Also in this case the peaks appear associated with strongly depleted signal modes with low intensities, and can be in different positions depending on the selected spatial structure. The bandwidth of non-classical variance $\mathcal{V}$ seems to be a general feature, almost independent of the structure selected. It corresponds to the FF region of intense tilted signal beams.

\section{Conclusions}

Non-linear optical systems present a wealth of physical phenomena including self-organizing spatial patterns and quantum correlations. Commonly employed methods to study these phenomena include the use of the positive $\mathrm{P}$ representation and the Wigner representation with third order derivatives neglected. Here we have investigated the use of the $\mathrm{Q}$ representation for studying quantum correlations in the DOPO at and above threshold. Positive diffusion is not guaranteed with the $\mathrm{Q}$ representation and this can lead to problems with divergent trajectories. For the DOPO, however, only positive diffusion occurs unless the fluctuations are strong enough to push the pump field up to twice the threshold value. This never occurred in our simulations and we have not attempted to calculate the effects of such highly unlikely trajectories on our ensembles [51.

Below threshold entanglement in quadratures and nonclassical intensity correlations are obtained, in agreement with linear analytical results. At threshold, a pair of quantum correlated twin beams is generated. These beams have wavevectors $\pm k_{c}$, corresponding to the critical wavelength. The quantum correlations are a natural consequence of the fundamental microscopic process in which a single pump photon is converted into a pair of signal photons. As we move further above threshold the twin beams can recombine to generate new pump photons. The combination of $\mathrm{a}+k_{c}$ and $\mathrm{a}-k_{c}$ photon regenerates one of the original pump photons. The combination of a pair of $+k_{c}$ photons or a pair of $-k_{c}$ photons, however, is a new process and introduces higher harmonics in the pump and thence in the the signal. Such processes can also degrade, but not completely suppress, the quantum correlations in some signal modes. This is a signature of the incoherent depletion of those modes. Yet further above threshold we enter a regime of spatially disordered structures. Remarkably, quantum correlations persist even in this regime, in the bandwidth of intense signal modes, where they take a form that depends on the spatial pattern that is generated.

We acknowledge financial support from the European Commission project QUANTIM (IST-2000-26019). RZ, $\mathrm{PC}$ and MSM acknowledge financial support from the Spanish MCyT projects PB97-0141-C02-02 and BFM 20001108. SMB thanks the Royal Society of Edinburgh and the Scottish Executive Education and Lifelong Learning Department for financial support.

\section{References}

1. L. A. Lugiato, M. Brambilla, and A. Gatti, in Advances in Atomic Molecular and Optical Physics 40, 229, B. Bederson and H. Walther eds. (Academic Press, New York, 1998).

2. M. I. Kolobov, Rev. Mod. Phys. 71, 1539 (1999).

3. Quantum Structures in nonlinear Optics and Atomic Physics, Focus Issue, Opt. Expr. 3, No. 2 (1998).

4. A. Gatti, E. Brambilla, L. A. Lugiato, and M. Kolobov, J. Opt. B: Quantum Semiclass. Opt. 2, 196 (2000).

5. G-L. Oppo, M. Brambilla, and L. A. Lugiato, Phys. Rev. A 49, 2028 (1994); G.-L. Oppo, M. Brambilla, D. Camesasca, A. Gatti, and L.A. Lugiato, J. Mod. Opt. 41, 1151 (1994).

6. M. Vaupel, A. Maître, and C. Fabre, Phys. Rev. Lett. 83, 5278 (1999); S. Ducci, N. Treps, A. Matre, and C. Fabre, Phys. Rev. A 64, 023803 (2001)

7. R. Graham, Phys. Rev. Lett. 52, 117 (1984).

8. A. Heidmann, R. J. Horowicz, S. Reynaud, E. Giacobino, C. Fabre and G. Camy, Phys. Rev. Lett. 59, 2555 (1987); A. Porzio, F. Sciarrino, A. Chiummo, M. Fiorentino, S. Solimeno, Opt. Comm. 194, 373 (2001).

9. K. Wiesenfeld, J. Stat. Phys. 38, 1071 (1985).

10. L. A. Lugiato, A. Gatti, H. Ritsch, I. Marzoli, and G. L. Oppo, J. Mod. Opt. 44, 1899 (1997).

11. I. Marzoli, A. Gatti, and L. A. Lugiato, Phys. Rev. Lett. 78, 2092 (1997).

12. Note that even far below threshold, where the critical mode does not play an important role, any two opposite modes $\mathbf{k}$ and $\mathbf{- k}$ are entangled.

13. A. Gatti, H. Wiedemann, L. A. Lugiato, I. Marzoli, G. L. Oppo, and S. M. Barnett, Phys. Rev. A 56, 877 (1997).

14. F. Castelli and L. A. Lugiato, J. Mod. Opt. 44, 765 (1997).

15. K. J. McNeil, and C. W. Gardiner, Phys. Rev. A 28, 1560 (1983); P. Kinsler, and P. D. Drummond, Phys. Rev. A 52, 783 (1995); P. D. Drummond, K. Dechoum, and S. Chaturvedi, Phys. Rev. A 65, 033806 (2002).

16. R. Zambrini, M. Hoyuelos, A. Gatti, P. Colet, L. Lugiato, and M. San Miguel, Phys. Rev. A 62, 63801 (2000).

17. A. Gatti and S. Mancini, Phys. Rev. A 65, 013816 (2002). 18. D. Gomila, and P. Colet, Phys. Rev. E (to be published). 
19. M. Santagiustina, P. Colet, M. San Miguel, and D. Walgraef, Phys. Rev. E 58, 3843 (1998).

20. R. Zambrini, S. M.Barnett, P. Colet and M. San Miguel, Phys. Rev. A 65, 023813 (2002).

21. R. Zambrini and M. San Miguel, Phys. Rev. A 66, 023807 (2002).

22. H. Carmichael, An Open Systems Approach to Quantum Optics, Lecture Notes in Physics, Vol. m18, (Springer, Berlin, 1993).

23. S. M. Barnett, and P. M. Radmore, Methods in Theoretical Quantum Optics, (Oxford Science Publications, Oxford 1997).

24. Here $\Delta_{i}$ and $a_{i}$ are cavity detunings and diffraction parameters for the pump and signal expressed in units of the associated decay constants $\gamma_{i}$

25. W. P. Schleich, Quantum Optics in Phase Space, (WileyVCH, Berlin, Germany, 2001).

26. C. W. Gardiner and P. Zoller, Quantum Noise Second Edition (Springer-Verlag, Berlin, 2000)

27. C.W. Gardiner, Stochastic Methods, (Springer, Berlin, 1985)

28. T. W. Marshall, Proc. R. Soc A, 276, 475 (1963)

29. P. D. Drummond and P. Kinsler, Quant. Semiclass. Opt. 7, 727 (1995); P. Kinsler, Phys. Rev. A 53, 2000 (1996); S. Chaturvedi and P. D. Drummond Phys. Rev. A 55, 912 (1997); D. T. Pope, P. D. Drummond, and W. J. Munro Phys. Rev. A 62, 042108 (2000).

30. P. D. Drummond and C. W. Gardiner, J. Phys. A: Math. Gen. 13, 2353 (1980).

31. The phase space doubling introduced with the $P_{+}$implies a much larger computational effort in the simulation of the Langevin equations. This is a critical problem with spatial degrees of freedom and justifies the search for alternative representations and methods.

32. A. Gilchrist, C. W. Gardiner and P. D. Drummond, Phys. Rev. A 55, 3014 (1997).

33. See U. Leonhardt, Measuring the Quantum State of Light, (Cambridge University Press, Cambridge, UK, 1997), Chapter VI and references therein.

34. R. F. Pawula, Phys. Rev. 162, 186 (1967).

35. See Appendix of: K. Vogel and H. Risken, Phys. Rev. A 38, 2409 (1988)

36. H. P. Yuen and P. Tombesi, Opt. Comm. 59, 155 (1986)

37. R. Zambrini and S. M. Barnett, Phys. Rev. A 65, 053810 (2002).

38. M. Bache, P. Scotto, R. Zambrini, M. San Miguel and M. Saffman, Phys. Rev. A 66, 013809 (2002).

39. When the condition for a positive diffusion (Eq. 14) is fulfilled, the arguments of the square roots in Eq. (16) are all positive.

40. S. Trillo, M. Haelterman, and A. Sheppard, Opt. Lett. 22, 970 (1997); N.B. Pettiaux, R.D. Li and P. Mandel, Opt. Commun. 72, 256 (1989); P.D. Drummond, K.J. McNeil, and D.F. Walls, Opt. Acta 27, 321 (1980).

41. C. Etrich, D. Michaelis, and F. Lederer, J. Opt. Soc. Am. B 19, 792 (2002).

42. D. Gomila, P. Colet, M. San Miguel, A. Scroggie and G.-L. Oppo, IEEE J. Quantum Elect. (to be published).

43. D. Gomila, P. Colet, M. San Miguel and G.-L. Oppo, Phys. Rev. Lett 87, 194101 (2001).

44. P. Coullet, C. Elphick and D. Rappaux, Phys. Rev. Lett. 65, 1352 (1990).
45. The shot noise level $\mathcal{N}_{X}$ for the variance of the quadrature superpositions is associated to the commutator of creation and destruction operators. A detailed evaluation of this quantity can be found in Ref. 20].

46. M. D. Reid and P. D. Drummond Phys. Rev. Lett. 60, 2731 (1988); Phys. Rev. A 40, 4493 (1989).

47. K. V. Kheruntsyan and K. G. Petrosyan Phys. Rev. A 62, 015801 (2000)

48. The deterministic steady state above threshold gives $\left|\alpha_{1}\left(k_{c}\right)\right|=\left|\alpha_{1}\left(-k_{c}\right)\right|$ and, with our choice of parameters, the phase sum $\theta_{+}+\theta_{-}=0$.

49. The transition depends effectively on the noise level. For example, for pump $E=1.1$, for which we have analyzed in Sect. 5.1 fluctuations in stripe patterns, a high level of noise $\left(g / \gamma=10^{-2}\right)$ leads to a disordered structure.

50. The discretization $\Delta k$ depends on the size of the system: $\Delta k=2 \pi / L=2 \pi / 4 \lambda_{c}$. With our choice of parameters $\Delta k=$ 0.075 .

51. Even very rare events, however, can have a profound influence on physical systems, so our procedure cannot be entirely satisfactory. A good example is found in laser cooling: F. Bardou, J.-P. Bouchaud, A. Aspect and C. CohenTannoudji, Lévy Statistics and Laser Cooling, (Cambridge University Press, Cambridge, 2002). 


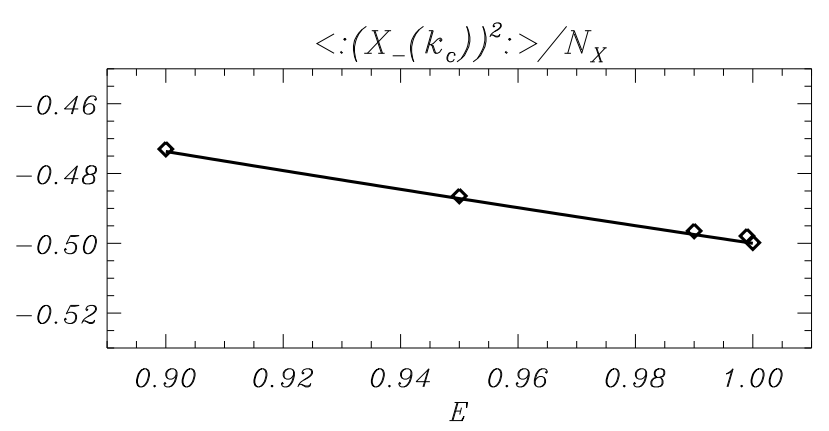

Fig. 2. Normal ordered variance of the damped quadrature $\hat{X}_{-}\left(k_{c}\right)$ normalized to shot noise: diamonds are results obtained by numerical simulation, while the continuous line corresponds to the analytical expression Eq. (26). For any trajectory at given pump intensity, we average during a time of $10^{7}$, integrating with a time discretization of $10^{-3}$ (with time scaled as in Eq. (11).

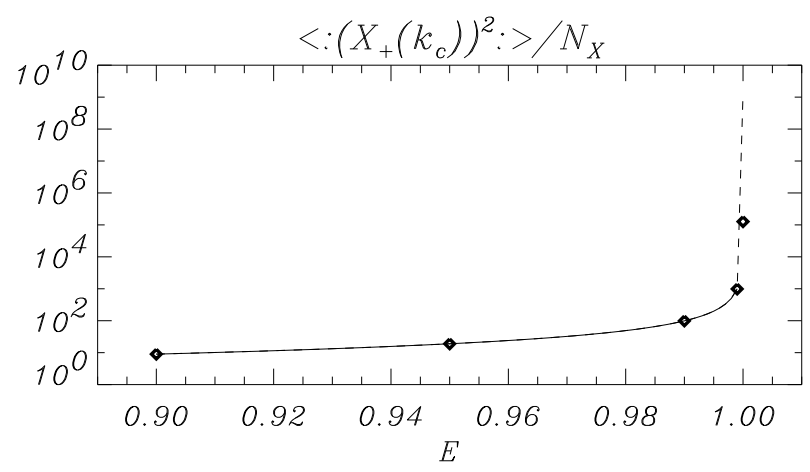

Fig. 3. Variance of the undamped quadrature $\hat{X}_{+}\left(k_{c}\right)$ : the diamonds are results obtained with numerical simulation, while the continuous line corresponds to the analytical expression Eq. (27). At the last point, corresponding to $E=1$, the linear treatment gives an infinite variance (the asymptotic behavior is represented by a dashed line), while our non-linear treatment gives the expected saturation.



Fig. 4. Analytical (continuous line) and numerical (diamonds) twin beams correlations $\mathcal{V}(k)$ (Eq. (28)) below threshold $(E=$ $0.99)$. The insert shows the mean intensity of the signal, with a maximum at $k_{c}=0.3$.

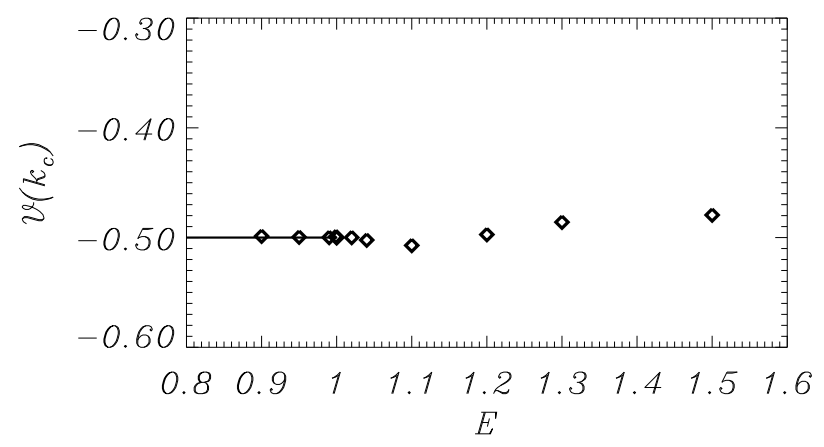

Fig. 5. Variance $\mathcal{V}\left(k_{c}\right)$ at the critical wave-vector $\left(k_{c}=0.3\right)$, increasing the pump intensity from below to above threshold $\left(E_{c}=1\right)$. The diamonds are numerical results and the continuous line is the analytical value obtained by linearizing below threshold.
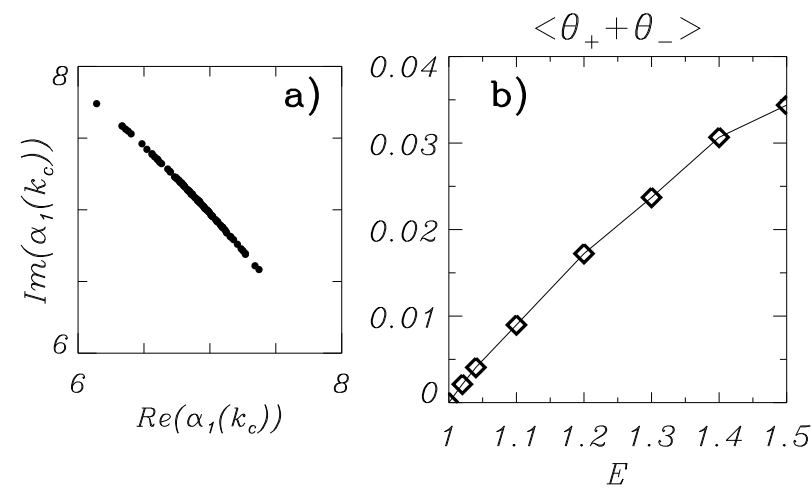

Fig. 6. a) Trajectory in the phase space of $\alpha_{1}\left(k_{c}\right)$ during $10^{7}$ scaled units for $E=1.02$. b) Phase sum $\left\langle\theta_{+}+\theta_{-}\right\rangle$(in radians) increasing the pump intensity.
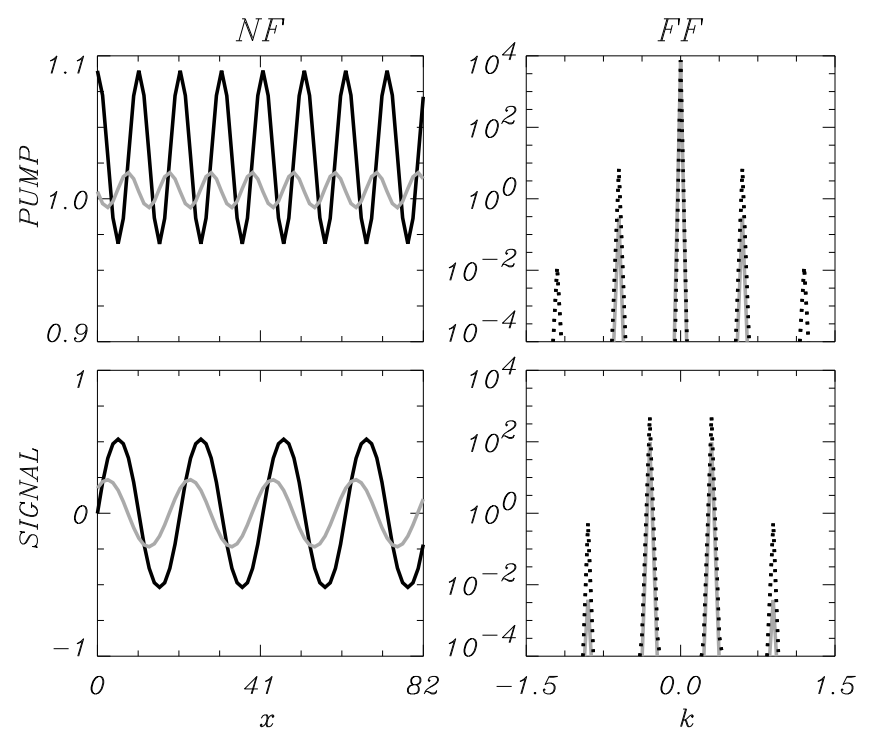

Fig. 7. Snapshot of the real part of the near fields (NF) and intensity (log scale) of the far fields (FF) for both the pump and the signal. The grey plots are obtained for a pump value $E=1.02$ and the black ones (in continuous or dotted lines) for $E=1.1$. 


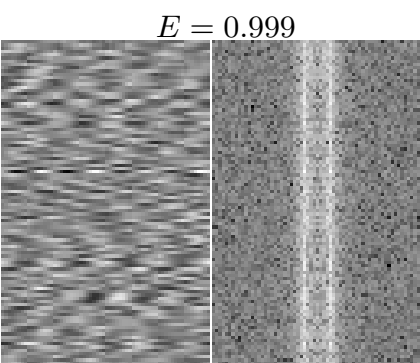

NF

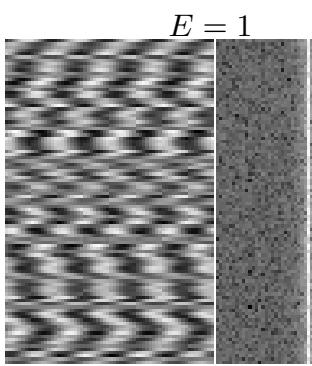

$\mathrm{NF}$

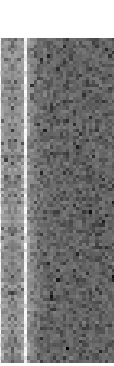

FF

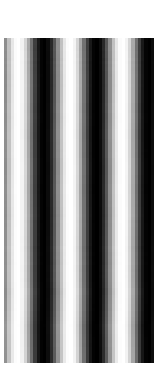

$\mathrm{NF}$

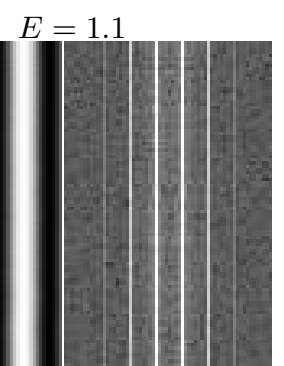

FF



FF

Fig. 1. Spatiotemporal evolution of the real part of the near field (NF) and of the intensity of the far field (FF) (in log scale), for different values of the pump $E=0.999,1,1.1,1.5$. The FF intensity is defined as $\left|\alpha_{i}(k)\right|^{2}$, where $\alpha_{i}(k)$ is the Fourier transform of the near field $\alpha_{i}(x)$. The horizontal coordinate is the transversal position ( $x$ in NF and $k$ in FF) describes by 64 points, and the vertical one is the time interval $10^{7}$ (in $\gamma$ units), using a discretization time step of $\Delta t=0.01$. The initial condition for the signal is $\alpha_{1}(x, 0)=10^{-5}\left(\epsilon(x)+10 \sin \left(k_{c} x\right)\right)$ with $\epsilon(x)$ Gaussian random numbers of variance one.

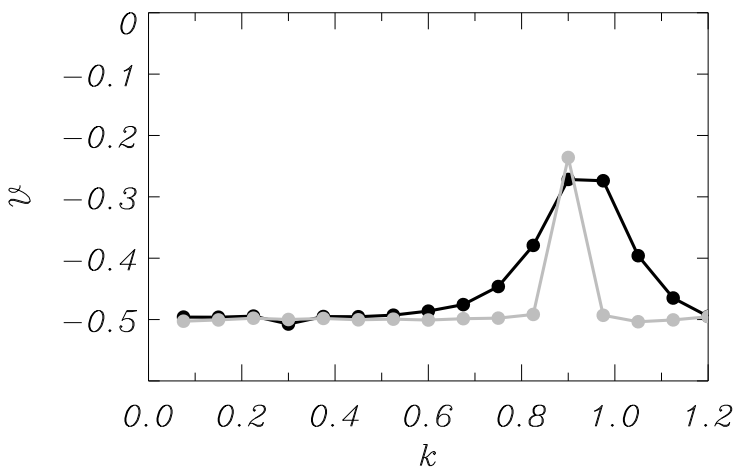

Fig. 8. Twin beams correlations $\mathcal{V}(k)$ above threshold, for $E=1.02$ (grey) and for $E=1.1$ (black).
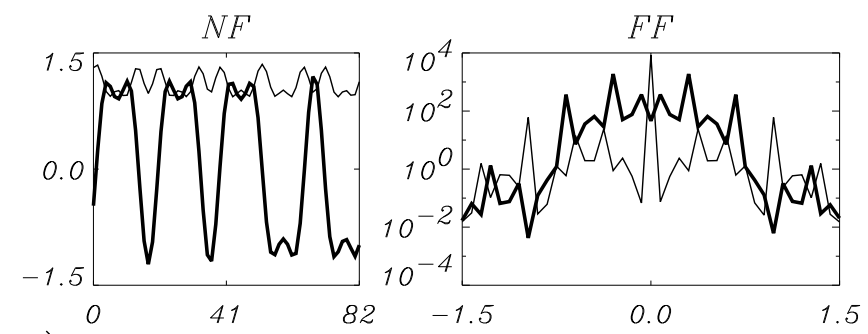

a)
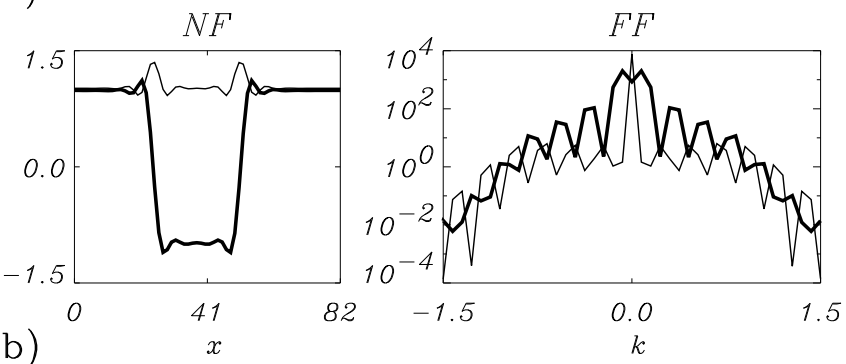

Fig. 9. Snapshots of the real part of the NF and intensity (log scale) of the FF for the pump (thin line) and the signal (thick line), for $E=1.5$, starting from a rolls pattern (a) and from a step function with values -1 and $+1(\mathrm{~b})$.

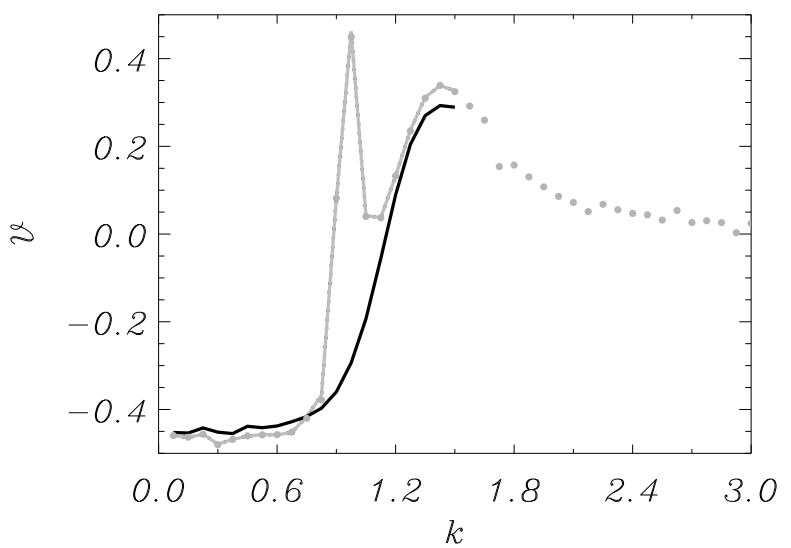

Fig. 10. Twin beams correlations $\mathcal{V}(k)$ for pump $E=1.5$. The grey (black) line is the spectrum $\mathcal{V}(k)$ for the pattern shown in Fig. 9 a (Fig. 9b ). The grey dotted line is obtained considering a system with the same size $\left(L=4 \lambda_{c}\right)$ but with a finer discretization (128 instead of 64 points), starting from a stripe configuration of critical wave-length. In this way we can see the asymptotic behavior of the spectrum for large wavevectors. For small wave-vectors the results are in good agreement with the simulation using 64 points. 

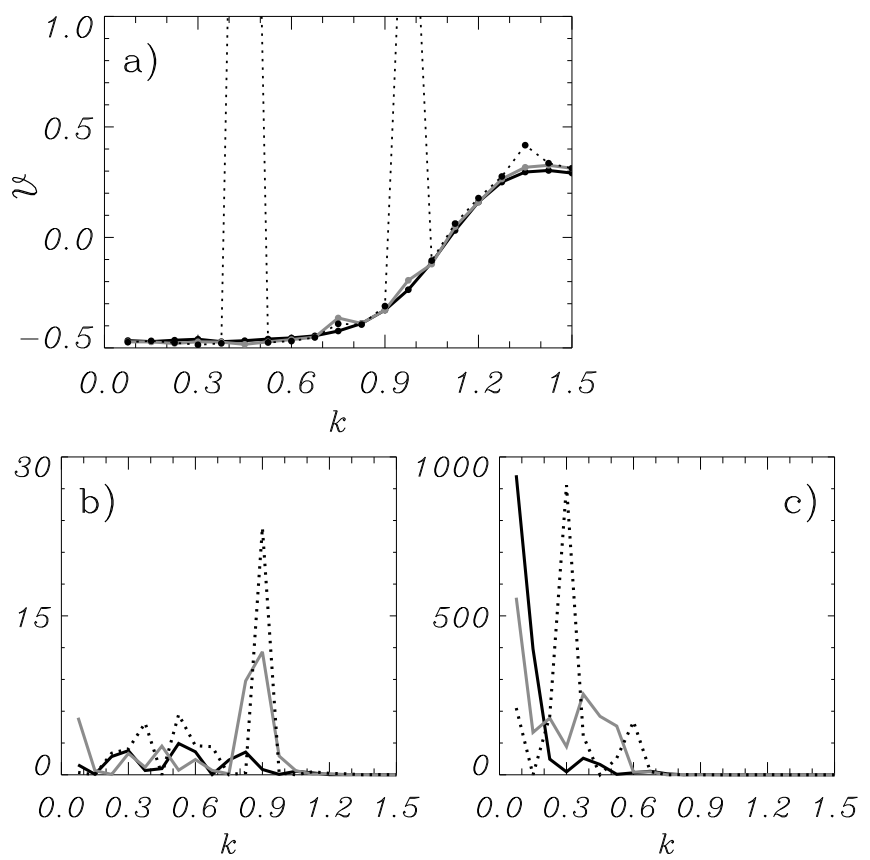

Fig. 11. a) Twin beams correlations $\mathcal{V}(k)$ for pump $E=1.3$, corresponding to different spatial structures, obtained starting from a step function (black line), from noise (grey line) and from a stripe pattern with critical wave-length (dotted line). Mean FF intensity in the pump $\left.\left(<N_{0}\right\rangle\right)$ (b) and in the signal $\left(<N_{1}>\right)$ fields (c), for the same three spatial configurations. 